\title{
Improved accuracy fullerene polarizability measurements in a long-baseline matter-wave interferometer
}

\author{
Yaakov Y. Fein $\odot$, Philipp Geyer, Filip Kiałka, Stefan Gerlich, and Markus Arndt $\odot^{*}$ \\ Faculty of Physics, University of Vienna, Boltzmanngasse 5, A-1090 Vienna, Austria
}

(Received 12 September 2019; published 9 December 2019)

\begin{abstract}
We present electric deflection results for the fullerenes $\mathrm{C}_{60}$ and $\mathrm{C}_{70}$ obtained with a long-baseline matterwave interferometer. The second grating of the interferometer is interchangeable between a material grating for fast atom beams and an optical phase grating for polarizable molecules. This allows us to use cesium as a calibration particle and thus measure molecular susceptibilities with improved systematic uncertainty. The static polarizabilities of $\mathrm{C}_{60}$ and $\mathrm{C}_{70}$ are measured as $4 \pi \varepsilon_{0} \times 87.4 \pm 0.4 \pm 2.5 \AA^{3}$ and $4 \pi \varepsilon_{0} \times 106.4 \pm 0.2 \pm 1.1 \AA^{3}$, respectively, in excellent agreement with previous deflection experiments, but with improved uncertainties.
\end{abstract}

DOI: 10.1103/PhysRevResearch.1.033158

\section{INTRODUCTION}

Electric deflectometry has been a hallmark of atomic and molecular beam research since the early work of Scheffer and Stark [1]. It has been employed in atomic [2], molecular [3-5], and cluster physics [6-8], as well as in the study of molecular dynamics [9] and in understanding the transition from atomic to bulk properties in clusters [10].

Matter-wave-assisted deflectometry is a natural extension of this technique, in which one measures the deflection of interference fringes rather than the deflection of an entire beam profile. Modern molecular interferometers [11-13] can resolve nanometer-scale fringe shifts, which provides orders of magnitude better resolution than classical beam deflectometry.

Several proof-of-principle experiments have demonstrated the technique by measuring the scalar static polarizability of fullerenes [14] as well as the dynamic susceptibilities of functionalized azobenzenes [15] and native vitamins [16]. The technique has also been used to identify the presence of electric dipole moments [17] and to distinguish molecular fragmentation pathways [18] and structural conformers [19].

Taking full advantage of the technique requires a good knowledge of the geometry of the deflection electrode as well as the various drift lengths of the experiment. An alternative approach, used here, is to calibrate the setup with an alkali atom, the polarizabilities of which have been accurately measured using far-field Mach-Zehnder interferometry [20-22].

Here, we use Talbot-Lau near-field interferometry [23,24], which is better suited to the typically low coherence of molecular beams than far-field techniques. We distinguish between two schemes, the Talbot-Lau interferometer (TLI)

\footnotetext{
*markus.arndt@univie.ac.at
}

Published by the American Physical Society under the terms of the Creative Commons Attribution 4.0 International license. Further distribution of this work must maintain attribution to the author(s) and the published article's title, journal citation, and DOI. with three equally spaced material gratings [25], and the Kapitza-Dirac-Talbot-Lau interferometer (KDTLI) in which the second grating is an optical phase grating [11].

The TLI scheme is well suited for fast particles with low polarizability, such as atoms, while the KDTLI scheme is best suited for slow and highly polarizable molecules. The measurements presented here were made in the Long-Baseline Universal Matter-Wave Interferometer (LUMI), which can alternate between the TLI and KDTLI schemes with nanometerprecise ultrahigh-vacuum compatible motors. Moreover, the 1-m grating separation provides a force sensitivity on the order of $10^{-26} \mathrm{~N}$. The universality of the interferometer allows for a direct comparison of atoms with molecules in the same apparatus.

We demonstrate the capabilities of LUMI by measuring the static scalar polarizability of the fullerenes $\mathrm{C}_{60}$ and $\mathrm{C}_{70}$ after calibration with cesium. The atomic calibration together with improved detection techniques allowed us to make both statistical and systematic uncertainty improvements compared to previous fullerene values $[4,14,26,27]$. These polarizability values are of interest due to the range of theoretical models which give conflicting polarizability predictions [28,29].

\section{EXPERIMENTAL DESIGN}

In a Talbot-Lau interferometer such as LUMI (see Fig. 1), the first grating prepares transverse coherence in the beam by acting as an array of point sources. Under partially coherent illumination, the second grating is self-imaged, imprinting revivals of the grating structure in the beam density. A third grating is placed at the position of one of these revivals and is transversely scanned to reveal a periodic modulation of the transmitted flux.

We use silicon nitride nanomechanical gratings with periods of $d=266 \mathrm{~nm}$ for the first and third gratings, while the second is interchangeable between another material grating and an optical phase grating formed by a retroreflected 532-nm laser $[11,25]$. The all-material TLI scheme was used for the cesium calibration, while the mixed material-optical KDTLI scheme was used for the fullerene measurements. 


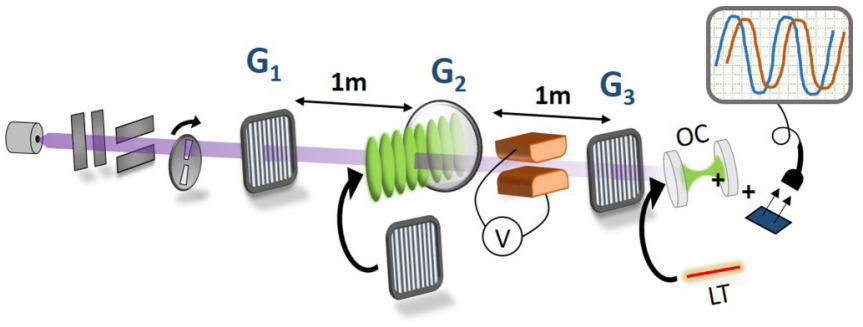

FIG. 1. Experimental setup, from left to right: the thermal source, beam collimators, chopper, and the three gratings $\left(\mathrm{G}_{1}-\mathrm{G}_{3}\right)$. $\mathrm{G}_{2}$ is interchangeable between a mechanical and an optical phase grating, and the electrode is between $\mathrm{G}_{2}$ and $\mathrm{G}_{3}$. The fullerenes are detected by thermal ionization in an optical cavity (OC) while the cesium atoms are counted after Langmuir-Taylor surface ionization (LT) and quadrupole mass selection (not shown).

We deflect the interference fringes with a specially designed electrode in which the value of $(\mathbf{E} \cdot \nabla) E_{x}$ is constant to within $1 \%$ over the traversed region [15]. An electric dipole moment is induced and experiences a constant force which yields a transverse phase shift of the fringes proportional to the static polarizability $\alpha_{\text {stat }}$,

$$
\Delta \phi=\alpha_{\text {stat }} \frac{K V^{2}}{m v^{2}}
$$

for a particle of mass $m$, velocity $v$, and with an applied voltage $V$. The constant $K$ depends on the length and longitudinal position of the electrode as well as the geometry of the electrode surface. For a force perfectly constant within the electrode and zero elsewhere, $K=\frac{2 \pi}{d}\left(L_{1}^{2} / 2-L_{1} L_{2}-L_{1} L\right) K_{E}$, where $K_{E}$ is the electrode geometry factor, $L$ the intergrating spacing $(0.98 \mathrm{~m}), L_{1}$ the distance from the second grating to the front edge of the electrode $(0.15 \mathrm{~m})$, and $L_{2}$ the electrode length $(0.04 \mathrm{~m})$.

The fringe patterns with amplitudes $A$ must be integrated over the velocity distribution $\rho(v)$ of the beam, giving the averaged pattern

$$
\bar{A} \cos (k x+\overline{\Delta \phi})=\int_{0}^{\infty} d v \rho A \cos (k x+\Delta \phi),
$$

where $x$ is the direction transverse to the grating bars, $k=$ $2 \pi / d$, and overbars denote velocity-averaged values. The averaged phase shift $\overline{\Delta \phi}$ which we measure is

$$
\overline{\Delta \phi}=\arg \left[\int_{0}^{\infty} d v \rho A \exp (i \Delta \phi)\right],
$$

where the velocity dependence of the amplitudes $A$ follows from the visibility function of the interferometer [30,31]. The velocity averaging reduces the fringe visibility for large deflections and finite velocity spreads.

\section{RESULTS}

\section{A. Cesium calibration}

We perform electric deflection of cesium to empirically determine the constant $K$ in Eq. (1), using the literature value for the polarizability of cesium, $\alpha_{\mathrm{Cs}}=4 \pi \varepsilon_{0} \times 59.39 \pm$
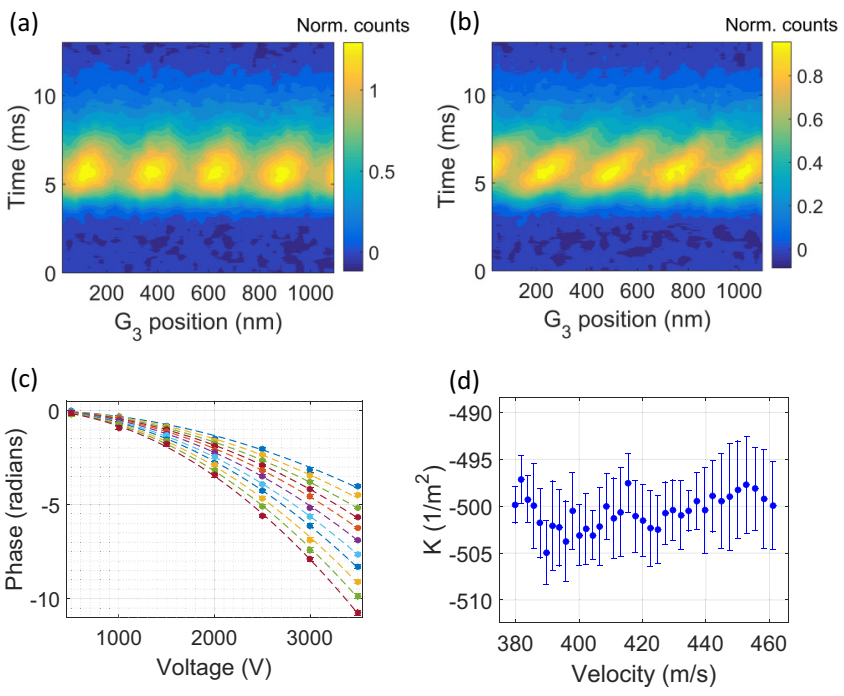

FIG. 2. (a) Time-resolved interference scan taken at the reference electrode voltage of $250 \mathrm{~V}$. (b) As (a) but for $2500 \mathrm{~V}$. (c) Cesium deflection curves for velocities ranging from 380 to $640 \mathrm{~m} / \mathrm{s}$, extracted from time-resolved data as shown in (a) and (b). (d) A selection of the curves in (c) is used to determine the mean calibration factor. Every fourth point is shown for clarity. Error bars are $68 \%$ confidence intervals of the fitted phase values.

$0.03 \pm 0.09 \AA^{3}$ [22], with the first error statistical and the second systematic.

Cesium was evaporated at $530 \mathrm{~K}$ and detected via Langmuir-Taylor surface ionization from a hot rhenium wire followed by quadrupole mass selection. For cesium we use the TLI scheme which gives visibilities up to $18 \%$ despite the dispersive van der Waals phase shift introduced at the second material grating $[11,32,33]$. The combination of high flux and efficient detection permitted the extraction of accurate phase data despite the moderate fringe visibility.

To estimate the statistical error of the calibration constant we compare the values of $K$ extracted for a range of beam velocities. A time-of-flight measurement was made at each position step of $\mathrm{G}_{3}$, yielding contour plots as shown in Fig. 2 . This method allows for the extraction of many individual interference scans at a broad range of velocities by taking line cuts at different times. Time-resolved interference measurements were taken for deflection voltages ranging from 500 to $3500 \mathrm{~V}$, with each position of the scan referenced to $250 \mathrm{~V}$ to remove the effect of slow phase drifts. Short-term stability of the interferometer was measured to be better than $0.03 \mathrm{~nm} / \mathrm{s}$.

The time of flight is measured by modulating the beam with a pseudorandom chopper and deconvoluting the time-resolved signal using the measured chopper sequence [34]. The velocity distribution at a given central velocity is approximated as a Gaussian with a full width at half maximum equal to the inherent resolution of the chopper, $\Delta t / t \approx 1 /\left(f N t_{\text {flight }}\right)$, where $f$ is the chopper rotation frequency and $N=255$ is the number of chopper bins. The extracted phase shifts in Fig. 2 for various velocities show the expected dependence on voltage.

Fitting Eq. (3) to the deflection data then yields the product $K \alpha_{\text {Cs }}$, which gives $K$ upon insertion of the literature value of 
$\alpha_{\mathrm{Cs}}$. The extracted values of $K$ are shown in Fig. 2(d), and an error-weighted average yields $K=500.42 \pm 0.18 \mathrm{~m}^{-2}$, where the uncertainty is the standard error of the values. The velocity dependence of the fringe amplitudes $A$ can be neglected here due to the small range of velocities contained in each deflection scan. From this value of $K$ the electrode calibration factor $K_{E}$ can also be estimated as $1.53 \times 10^{4} \mathrm{~m}^{-3}$.

With this empirical value of the calibration factor $K$, we can now extract the polarizability of other particles in the same setup.

\section{B. Fullerene measurements}

A thermal fullerene beam $\left(\mathrm{C}_{60}: 843 \mathrm{~K} ; \mathrm{C}_{70}: 880 \mathrm{~K}\right)$ was produced from a ceramic oven, and velocities were gravitationally selected with the aid of several vertical delimiters (down to $100 \mu \mathrm{m}$ aperture height). The lower beam velocity compared to cesium made the velocity selection more effective, allowing us to directly tune the velocity distribution without the need for time-resolved interference measurements. The molecules were detected via thermal ionization $[35,36]$ in a 532-nm optical cavity providing up to $300 \mathrm{~W}$ of intracavity power in a beam waist of $50 \mu \mathrm{m}$. The ions were then directly counted with an electron multiplier. Mass selection was unnecessary due to the purity of the samples $\left(\mathrm{C}_{60} 98 \%, \mathrm{C}_{70}>99 \%\right)$ and the negligible dark count rate with thermal ionization. The isotopic distribution of the fullerenes is accounted for by using the molar mass in the calculation of the polarizability. The cavity-enhanced thermal ionization scheme was particularly beneficial since it is more efficient than the electron impact and also acts as a vertical delimiter, thus reducing the velocity spread of the detected beam.

Given the low-beam velocity and high polarizability of the fullerenes we employ the KDTLI scheme, thus avoiding dephasing due to van der Waals interactions at the second grating. Interference visibilities of $35 \%$ and $24 \%$ could be obtained for $\mathrm{C}_{60}$ and $\mathrm{C}_{70}$, respectively.

Interference scans were collected for electrode voltages of $250-3250 \mathrm{~V}$ for a range of selected beam velocities, as
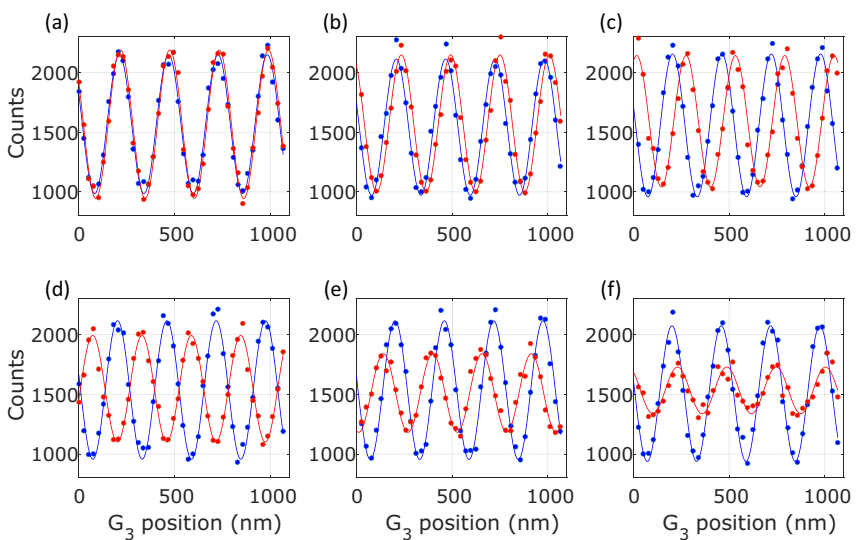

FIG. 3. (a)-(f) Deflection data for $\mathrm{C}_{60}$ with sine fits for increasing deflection voltages from 500 to $3000 \mathrm{~V}$ in steps of $500 \mathrm{~V}$. Red curves are with the deflection voltage applied, blue with a fixed $250 \mathrm{~V}$ reference voltage. The mean velocity for these measurements was $221 \mathrm{~m} / \mathrm{s}$. The fringe deflection and visibility loss due to velocity averaging are clearly visible. (a)

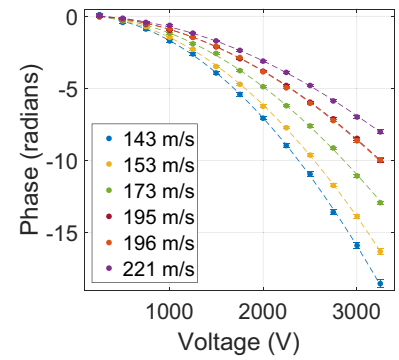

(c)
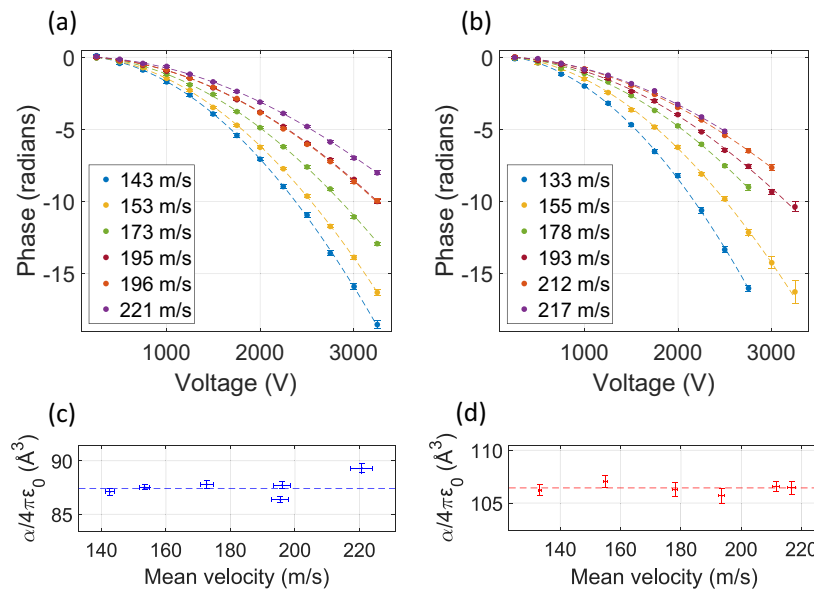

(d)

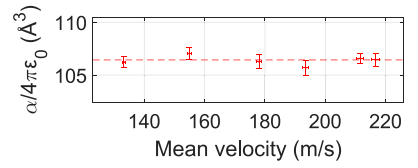

FIG. 4. (a) $\mathrm{C}_{60}$ deflection curves for a range of mean velocities. (b) $\mathrm{C}_{70}$ deflection curves. (c) $\mathrm{C}_{60}$ extracted polarizability values for the various velocities with error bars corresponding to $68 \%$ confidence intervals of the fitted phases and the mean velocity. (d) As (c), but for $\mathrm{C}_{70}$. The smaller horizontal error bars are because more data were taken for the velocity calibration during the $\mathrm{C}_{70}$ data run.

illustrated in Fig. 3, with the complete deflection data shown in Fig. 4. Velocity measurements were taken six times both before and after each deflection measurement of a given velocity class. A reference measurement at $250 \mathrm{~V}$ was taken at each position step to compensate for any phase drifts. The power of the 532-nm laser creating the standing light wave was adjusted for each velocity class, and ranged from 4.4 to $9.3 \mathrm{~W}$.

Inserting the geometry constant $K$ obtained from the cesium measurements into Eq. (3) gives the mean polarizability values $4 \pi \varepsilon_{0} \times 87.4 \pm 0.4 \pm 2.5 \AA^{3}$ and $4 \pi \varepsilon_{0} \times 106.4 \pm$ $0.2 \pm 1.1 \AA^{3}$ for $\mathrm{C}_{60}$ and $\mathrm{C}_{70}$, respectively. The first uncertainty (statistical) is estimated from the standard error of the extracted polarizabilities for different velocity classes as shown in Figs. 4(c) and 4(d). The second uncertainty (systematic) is dominated by the uncertainty of the velocity measurement calibration and a smaller contribution due to the sample impurity. The velocity dependence of the fringe amplitudes $A$ is taken into account in Eq. (3) by using the visibility function in Ref. [31]. A small correction has been applied to account for the slightly different longitudinal position of the optical grating used here and the material grating used for the cesium calibration. Our measured polarizability values are in excellent agreement with previous results, which range from $4 \pi \varepsilon_{0} \times 76.5 \AA^{3}$ to $4 \pi \varepsilon_{0} \times 88.9 \AA^{3}$ for $\mathrm{C}_{60}$ and from $4 \pi \varepsilon_{0} \times 101.9 \AA^{3}$ to $4 \pi \varepsilon_{0} \times 108.5 \AA^{3}$ for $\mathrm{C}_{70}[4,14,26,27]$.

\section{SUMMARY}

The LUMI experiment permits the direct comparison of atoms and molecules across a wide range of masses and polarizabilities. This allowed us to calibrate the deflection setup with cesium and then measure the static dipole polarizability of the fullerenes $\mathrm{C}_{60}$ and $\mathrm{C}_{70}$, with a systematic uncertainty two times better for $\mathrm{C}_{60}$ and more than five times better for $\mathrm{C}_{70}$. 


\section{ACKNOWLEDGMENTS}

This project has received funding from the European Research Council (ERC) under the European Union's Horizon
2020 research and innovation program (Grant No. 320694), and the Austrian Science Fund (FWF) within program P30176 and W1210-N25.
[1] H. Scheffers and J. Stark, Phys. Z. 35, 625 (1934).

[2] L. Ma, J. Indergaard, B. Zhang, I. Larkin, R. Moro, and W. A. de Heer, Phys. Rev. A 91, 010501(R) (2015).

[3] R. Antoine, I. Compagnon, D. Rayane, M. Broyer, P. Dugourd, G. Breaux, F. C. Hagemeister, D. Pippen, R. R. Hudgins, and M. F. Jarrold, Eur. Phys. J. D 20, 583 (2002).

[4] R. Antoine, P. Dugourd, D. Rayane, E. Benichou, M. Broyer, F. Chandezon, and C. Guet, J. Chem. Phys. 110, 9771 (1999).

[5] R. Moro, R. Rabinovitch, C. Xia, and V. Kresin, Phys. Rev. Lett. 97, 123401 (2006).

[6] S. Schäfer and R. Schäfer, Phys. Rev. B 77, 205211 (2008).

[7] S. Schäfer, S. Heiles, J. Becker, and R. Schäfer, J. Chem. Phys. 129, 044304 (2008)

[8] W. D. Knight, K. Clemenger, W. A. de Heer, and W. A. Saunders, Phys. Rev. B 31, 2539 (1985).

[9] D. Rayane, R. Antoine, P. Dugourd, E. Benichou, A. R. Allouche, M. Aubert-Frécon, and M. Broyer, Phys. Rev. Lett. 84, 1962 (2000).

[10] J. Bowlan, A. Liang, and W. A. de Heer, Phys. Rev. Lett. 106, 043401 (2011).

[11] S. Gerlich et al., Nat. Phys. 3, 711 (2007).

[12] P. Haslinger, N. Dörre, P. Geyer, J. Rodewald, S. Nimmrichter, and M. Arndt, Nat. Phys. 9, 144 (2013).

[13] Y. Y. Fein, P. Geyer, P. Zwick, F. Kiałka, S. Pedalino, M. Mayor, S. Gerlich, and M. Arndt, Nat. Phys. (2019), doi: 10.1038/s41567-019-0663-9.

[14] M. Berninger, A. Stefanov, S. Deachapunya, and M. Arndt, Phys. Rev. A 76, 013607 (2007).

[15] M. Gring et al., Phys. Rev. A 81, 031604(R) (2010).

[16] L. Mairhofer, S. Eibenberger, J. P. Cotter, M. Romirer, A. Shayeghi, and M. Arndt, Angew. Chem., Int. Ed. 56, 10947 (2017).

[17] S. Eibenberger, S. Gerlich, M. Arndt, J. Tüxen, and M. Mayor, New J. Phys. 13, 043033 (2011).

[18] S. Gerlich, M. Gring, H. Ulbricht, K. Hornberger, J. Tüxen, M. Mayor, and M. Arndt, Angew. Chem., Int. Ed. 47, 6195 (2008).
[19] J. Tüxen, S. Gerlich, S. Eibenberger, M. Arndt, and M. Mayor, Chem. Commun. 46, 4145 (2010).

[20] A. Miffre, M. Jacquey, M. Büchner, G. Trenec, and J. Vigue, Phys. Rev. A 73, 011603(R) (2006).

[21] W. F. Holmgren, M. C. Revelle, V. P. A. Lonij, and A. D. Cronin, Phys. Rev. A 81, 053607 (2010).

[22] M. D. Gregoire, I. Hromada, W. F. Holmgren, R. Trubko, and A. D. Cronin, Phys. Rev. A 92, 052513 (2015).

[23] J. F. Clauser and S. Li, Phys. Rev. A 49, R2213 (1994).

[24] B. Brezger, M. Arndt, and A. Zeilinger, J. Opt. B 5, S82 (2003).

[25] B. Brezger, L. Hackermüller, S. Uttenthaler, J. Petschinka, M. Arndt, and A. Zeilinger, Phys. Rev. Lett. 88, 100404 (2002).

[26] I. Compagnon, R. Antoine, M. Broyer, P. Dugourd, J. Lermé, and D. Rayane, Phys. Rev. A 64, 025201 (2001).

[27] A. Ballard, K. Bonin, and J. Louderback, J. Chem. Phys. 113, 5732 (2000).

[28] K. Bonin and V. Kresin, Electric-Dipole Polarizabilities of Atoms, Molecules and Clusters (World Scientific, Singapore, 1997).

[29] D. S. Sabirov, RSC Adv. 4, 44996 (2014).

[30] S. Nimmrichter and K. Hornberger, Phys. Rev. A 78, 023612 (2008).

[31] K. Hornberger, S. Gerlich, H. Ulbricht, L. Hackermüller, S. Nimmrichter, I. Goldt, O. Boltalina, and M. Arndt, New J. Phys. 11, 043032 (2009).

[32] R. E. Grisenti, W. Schöllkopf, J. P. Toennies, G. C. Hegerfeldt, and T. Köhler, Phys. Rev. Lett. 83, 1755 (1999).

[33] C. Brand et al., Nat. Nanotechnol. 10, 845 (2015).

[34] D. D. Koleske and S. J. Sibener, Rev. Sci. Instrum. 63, 3852 (1992).

[35] D. Ding, J. Huang, R. N. Compton, C. E. Klots, and R. E. Haufler, Phys. Rev. Lett. 73, 1084 (1994).

[36] O. Nairz, M. Arndt, and A. Zeilinger, J. Mod. Opt. 47, 2811 (2000). 